\title{
THE EXISTENCE OF HOMOMORPHISMS IN COMPACT CONNECTED ABELIAN GROUPS
}

\author{
GERALD L. ITZKOWITZ ${ }^{1}$
}

1. Introduction. Andre Weil [4] has shown that for an Abelian topological group $G$, an open divisible subgroup $H \subset G$ is topologically a direct summand of $G$. It is then immediate that with Abelian groups each divisible group is a direct summand (algebraically) of every group in which it is contained (give the groups in question the discrete topology making them into topological groups). We will use this observation to deduce an existence theorem for homomorphisms between groups. Then making use of some results of Braconnier [1], we give a necessary and sufficient condition for the existence of continuous nonzero homomorphisms between a compact connected Abelian topological group and each compact connected Abelian group.

The motivation for this work is an example due to $\mathrm{W}$. Comfort of a pair $G_{1}, G_{2}$ of compact connected Abelian groups for which there is no nonzero continuous homomorphism of $G_{1}$ into $G_{2}$. In the example, $G_{1}=$ circle, and $G_{2}=\left(Q_{d}\right)^{\wedge}$, where $Q_{d}$ denotes the rationals with the discrete topology. The example works because the elements of finite order in $G_{1}$ are dense in $G_{1}$ while there are no elements of finite order in $G_{2}$. This leads to the conjecture that we can characterize the existence of continuous homomorphisms between compact connected Abelian topological groups in terms of the elements of finite order in the groups. We will show that this is indeed the case (see Theorem 3 ).

\section{The existence theorem and its applications.}

Theorem 1 (Existence). Let $G$ be a torsion free Abelian group. $A$ necessary and sufficient condition for the existence of a nonzero homomorphism from each torsion free Abelian group $H$ into $G$ is that $G$ contain a nontrivial divisible subgroup $D$, so that $G$ is the direct sum $D \times G / D$.

Proof. Observe that if $x \in H$, and if $0 \neq y \in D$ then the function $\phi$ defined by $\phi(n x)=n y, n \in Z=$ integers, extends to a homomorphism $\phi^{\prime}$ of $H$ into $D$, since $D$ is divisible (Kaplansky [3]).

Conversely, suppose that $G$ contains no divisible subgroups. Then

Received by the editors October 31, 1966.

1 The author gratefully acknowledges support by the National Science Foundation under Grant GP-6327. 
if $H$ is divisible, $G$ contains no nonzero homomorphic image of $H$ since the homomorphic image of a divisible Abelian group is divisible.

In what follows $G$ will denote a compact connected Abelian topological group, $B$ will denote the subgroup of elements of finite order in $G$, and $B^{-}$will denote its topological closure in $G$.

TheOREm 2. $B^{-}$is a topological direct summand of $G$, so that $G=B^{-}$ $\times G / B^{-}$.

Proof. According to 25.30 (b) of [2], it suffices to show that $G / B^{-}$ is torsion free.

We show first that $B^{-}$is divisible. Let $x \in B^{-}$, let $n$, a positive integer, be given, and let $\left\{x_{\alpha}\right\} \subset B$ be a net converging to $x$. Since the elements of finite order form a divisible group it follows that for each $\alpha$ there is a $y_{\alpha} \in B$ satisfying $n y_{\alpha}=x_{\alpha}$. Furthermore, since $G$ is compact $B^{-}$is compact and hence $\left\{y_{\alpha}\right\}$ has a convergent subnet $\left\{y_{\beta}\right\}$ converging to $y \in B^{-}$. Obviously

$$
n y=\lim _{\beta} n y_{\beta}=\lim _{\alpha} n y_{\alpha}=\lim _{\alpha} x_{\alpha}=x .
$$

Thus $B^{-}$is divisible.

Since $B^{-}$is divisible it is algebraically a direct summand of $G$. Thus if $x \notin B^{-}$we have $x=x_{1}+x_{2}$ where $x_{1} \in B^{-}$and $x_{2} \in\left(B^{-}\right)^{\prime}$, the algebraic complement of $B^{-}$. Thus if $n x \in B^{-}$then $n x_{2} \in B^{-}$. Clearly this can happen if and only if $n x_{2}=0$. Thus $x_{2}$ has finite order and hence is in $B$, a contradiction. This proves that $G / B^{-}$is torsion free, and the theorem is proved.

Braconnier [1], has shown that if $G_{1}$ and $G_{2}$ are locally compact Abelian groups and if $\phi$ is an open continuous homomorphism of $G_{1}$ in to $G_{2}$ then $\tilde{\phi}$, defined by $\tilde{\phi}(\chi)=\chi \circ \phi$, is an open continuous homomorphism of $\hat{G}_{2}$ (the character group of $G_{2}$ ) into $A\left(\phi^{-1}\{0\}\right)$ (the annihilator of the set $\left.\phi^{-1}\{0\}\right)$. We will use this in the proof of our next result.

TheOREM 3. In order that the compact connected Abelian group $G$ admit a nonzero continuous homomorphism into each nonzero compact connected Abelian group, it is necessary and sufficient that the torsion subgroup of $G$ not be dense in $G$.

Proof. We make the following observation. If $H$ is a compact connected Abelian group then it is well known that $\hat{H}$, the character group of $H$ is discrete and torsion free.

By Theorem 1, there exists a nonzero continuous homomorphism $h$ from each $\hat{H}$ that is discrete and torsion free in to $\hat{G}$ if and only if $\hat{G}$ 
is topologically isomorphic with $D \times \hat{G} / D$ where $D$ is divisible and nontrivial. By the theorem of Braconnier quoted above the homomorphism $\tilde{h}$ is nonzero and continuous from $G$ in to $H$ if and only if $h$ is nonzero from $\hat{H}$ into $\hat{G}$. Furthermore it is well known for discrete Abelian groups $D$, that $D$ is divisible if and only if $\hat{D}$ is torsion free. Obviously $\hat{D}$ is nonzero in the present case, so that the torsion subgroup of $G$ is not dense in $G$. The converse is immediate from Theorem 2 .

\section{BiBLIOGRAPHY}

1. J. Braconnier, Sur les groupes topologiques localement compacts, J. Math. Pures Appl. 27 (1948), 1-85.

2. E. Hewitt and K. A. Ross, Abstract harmonic analysis, Vol. 1, Springer-Verlag, Heidelberg, 1963.

3. I. Kaplansky, Infinite Abelian groups, Univ. of Michigan Press, Ann Arbor, 1954.

4. A. Weil, L'integration dans les groupes topologiques et ses applications, Actualités Sci. Ind. Nos. 869, 1145, Hermann, Paris, 1941 and 1951.

State University of New York at Buffalo 